\title{
LA VIGILANCIA DE LOS EVENTOS SANITARIOS A TRAVÉS DE LOS MEDIOS DE COMUNICACIÓN EN PERÚ
}

\author{
Ada Palpan-Guerra1,a, César V. Munayco ${ }^{1, b, c}$
}

\begin{abstract}
RESUMEN
Objetivo. Caracterizar los eventos y brotes detectados por la vigilancia de eventos sanitarios a través de medios de comunicación entre los años 2013 y 2015 . Además, determinar la proporción de eventos o brotes que no fueron captados por los sistemas de vigilancia regulares. Materiales y métodos. Se analizaron los datos registrados por la vigilancia de eventos sanitarios a través de medios de comunicación del Centro Nacional de Epidemiologia y Control de Enfermedades (CDC). Esta vigilancia registra diariamente las noticias de los medios de comunicación escritos, radio, televisión e Internet del país, las clasifica por tipo de evento, determina si el evento fue registrado, investigado y confirmado, además, de evaluar si la noticia fue alarmista, informativa o educativa. Resultados. Se notificó que el $49,4 \%$ de eventos que no habían sido identificados por los sistemas de vigilancia epidemiológica a nivel local, regional o nacional. Además, el $17,4 \%$ de las noticias analizadas fueron alarmistas. Conclusión. Este tipo de vigilancia es una herramienta con un alto potencial para identificar brotes y/o eventos no identificados por los sistemas de vigilancia regulares, y deben de fortalecerse, además de usar sus resultados para mejorar la toma de decisiones.
\end{abstract}

Palabras clave: Vigilancia; Medios de comunicación (fuente: DeCS BIREME).

\section{THE ROLE OF MEDIA-BASED SURVEILLANCE SYSTEM FOR PUBLIC HEALTH EVENTS IN PERU}

\begin{abstract}
Objectives. The objective of this study was to describe events and outbreaks identified by Media-Based Surveillance System for Public Health Events between 2013 and 2015. Further, to determine the percentage of events or outbreaks that were not identifing by regular surveillance systems. Materials and methods. We analyzed the data collected from Media-Based Surveillance System for Public Health Events of the National Center for Epidemiology and Disease Control. This surveillance system records news from the written media, radio, television, and Internet in Peru on a daily basis; classifies them according to the type of event; determines whether the event was registered, investigated, and confirmed; and evaluates whether the news was alarmist, informative, or educational. Results. Overall, $49.4 \%$ of the events reported had not been identified by epidemiological surveillance systems at the local, regional, or national level. In addition, $17.4 \%$ of the analyzed news was alarmist. Conclusion. This type of surveillance has a high potential to identify outbreaks and/or health events not identified by regular surveillance systems and should thus be strengthened. In addition, the results of this tool may help to improve decision-making.
\end{abstract}

Key words: Surveillance, Media (source: MeSH NLM).

\section{INTRODUCCIÓN}

Con la finalidad de proteger la salud pública, previniendo la diseminación de enfermedades, el Reglamento Sanitario Internacional (RSI) ha ampliado la notificación usual de enfermedades infecciosas, incluyendo a la vigilancia de eventos de salud pública de orígenes diversos dentro de su arsenal; e insta a los estados miembros a fortalecer la capacidad de sus sistemas de vigilancia para detectar, evaluar, notificar y responder a todos los eventos de salud o riesgos para la salud que pueden constituir una amenaza para la salud humana ${ }^{(1,2)}$.
Además, el RSI establece que para las tareas de vigilancia y respuesta, los países preparen y refuercen sus capacidades básicas en comunicación de riesgos y vigilancia basada en eventos, para hacer frente a epidemias $y / 0$ emergencias de salud pública que signifiquen un peligro nacional y/o internacional; el cual contribuirá a controlar lo más rápido posible las repercusiones para la salud en cualquiera de las etapas de una emergencia o desastre ${ }^{(2)}$.

Por otro lado, los componentes de alerta temprana de los sistemas de vigilancia convencionales dependen

Centro Nacional de Epidemiología, Prevención y Control de Enfermedades, Ministerio de Salud. Lima, Perú.

Licenciada en Comunicación Social, ${ }^{\mathrm{b}}$ médico, ${ }^{\mathrm{c}}$ doctor en Salud Pública.

Recibido: 16/05/2017 Aprobado: 20/09/2017 En línea: 29/09/2017

Citar como: Palpan-Guerra A, Munayo CV. La vigilancia de los eventos sanitarios a través de los medios de comunicación en el Perú. Rev Peru Med Exp Salud Publica. 2017;34(3):395-403. doi: 10.17843/rpmesp.2017.343.2879 
principalmente de la información recopilada en los establecimientos de salud. Este mecanismo de alerta temprana debe ser sensible para detectar y responder rápidamente a las alertas que provengan de los sistemas de vigilancia dentro y fuera del sector de la salud. En muchos casos, estos mecanismos de alerta basados en la notificación de casos son insuficientes, debido al retraso que tienen estos sistemas desde la notificación hasta el momento en que esté disponible para el análisis y, porque, en su mayoría, son sistemas de notificación semanal ${ }^{(1)}$.

La Organización Mundial de la Salud (OMS) reconoce a los medios de comunicación como el canal más importante de difusión masiva de información durante una emergencia de salud pública, ya que proporcionan información decisiva para la gestión eficaz de las comunicaciones en una situación de brote epidémico, debido a que la globalización y las nuevas tecnologías de la comunicación hacen que las noticias viajen en tiempo real ${ }^{(1,3)}$.

La vigilancia basada en eventos contempla mecanismos de vigilancia y monitoreo de los medios de comunicación masiva como radio, televisión, prensa escrita e internet, como una estrategia que permita la detección temprana de potenciales brotes epidémicos y otras emergencias sanitarias, que pueden representar el inicio de una emergencia con impacto en la salud pública ${ }^{(4,6)}$.

El Centro Nacional de Epidemiología, Prevención y Control de Enfermedades (CDC) del Ministerio de Salud del Perú (MINSA) ha incorporado diferentes subsistemas de vigilancia como parte del Sistema de Alerta y Respuesta (SAR) que conduce (Noti-SP, vigilancia de enfermedades, vigilancia sindrómica en hospitales centinela, vigilancia de eventos sanitarios en medios de comunicación, resultados de laboratorio, entre otros) ${ }^{(7,8)}$.

EI SAR procura aumentar la sensibilidad para la detección, la calidad de la evaluación de riesgos, y la oportunidad y eficacia de la respuesta a los riesgos para la salud pública, a modo de reducir al mínimo las consecuencias negativas para la salud de la población afectada ${ }^{(1)}$. En ese sentido, el objetivo principal del SAR es identificar precozmente la ocurrencia de riesgos, brotes o emergencias sanitarias a fin de implementar acciones de prevención y/o control ${ }^{(9)}$.

En ese marco, la vigilancia de eventos sanitarios en medios de comunicación cumple con proporcionar los insumos para investigar posibles sucesos que luego, una vez evidenciados o comprobados, deben ser investigados ya sea como brotes, epidemias, epizootias u otras emergencias sanitarias ${ }^{(7)}$. Se constituye así, en una de las estrategias más sensibles de las que hace uso el CDC del MINSA.

\section{MENSAJES CLAVE}

Motivación para realizar Los medios de comunicación son el canal más importante de difusión masiva de información durante una emergencia sanitaria. Pretendemos caracterizar los eventos detectados por la vigilancia de eventos sanitarios (ES) en medios de comunicación (2013 y 2015).

Principales hallazgos. El estudio confirmó la importancia de la vigilancia de ES en medios de comunicación. Se identificó que el $49,4 \%$ de los ES fueron difundidos primero por los medios de comunicación y luego verificados y confirmados por los sistemas de vigilancia epidemiológica.

Implicancias. La vigilancia de ES a través de los medios de comunicación permitió identificar casi el $50 \%$ de los eventos que no habían sido identificados por los sistemas de vigilancia convencionales.

El inicio de la vigilancia de eventos sanitarios en medios de comunicación para detectar brotes, epizootias y otras emergencias sanitarias data desde la creación de la Oficina y luego Dirección General de Epidemiología (DGE), y con el transcurso de los años esta vigilancia ha sufrido adaptaciones y mejoras en sus procesos y metodología, permitiendo que sea más sensible y dinámica en cuanto a la detección, registro, notificación y verificación del evento sanitario o brote ${ }^{(8)}$.

Esta vigilancia de eventos sanitarios en medios de comunicación tiene como objetivo: detectar oportunamente los brotes, epizootias y otros eventos de importancia para la salud pública con el fin de incorporarlos en el sistema de vigilancia y así contribuir en el control de problemas de salud, los cuales pasan por un proceso de verificación y así confirmar o descartar el evento difundido por los medios de comunicación ${ }^{(8-10)}$.

Como se ha mencionado, la vigilancia de eventos sanitarios en medios de comunicación es una estrategia complementaria de los sistemas de vigilancia basado en notificación de casos, en ese sentido, el objetivo de este manuscrito es caracterizar los eventos y brotes detectados por este sistema entre los años 2013 y 2015.

\section{MATERIALES Y MÉTODOS}

Se realizó un estudio retrospectivo de la base de datos de la vigilancia de eventos sanitarios en medios de comunicación del Centro Nacional de Epidemiología, Prevención y Control de Enfermedades (antes DGE) del MINSA.

\section{DESCRIPCIÓN DEL SUBSISTEMA DE VIGILANCIA DE EVENTOS SANITARIOS EN MEDIOS DE COMUNICACIÓN}

En el marco de la vigilancia de eventos sanitarios en medios de comunicación, la búsqueda diaria de todas las noticias relacionadas a brotes, epizootias, y otros eventos de importancia para la salud pública se realizó 
en las noticias publicadas en la prensa escrita, televisiva, radial e internet de cobertura nacional y regional, durante el periodo 2013 - 2015. Las noticias son registradas en el aplicativo de la vigilancia alojado en la intranet de la página web del CDC.

El registro de las noticias se realizateniendoen consideración lo siguiente: el primer medio de comunicación que difundió el evento o en el que fuera detectado primero durante la vigilancia era registrado como la fuente de información primaria, no se consideró en el registro otros medios de comunicación que difundieron la misma noticia posterior al registro inicial. Se revisaron las noticias publicadas por cinco diarios de cobertura nacional (prensa escrita y web): El Comercio, Correo, La República, Perú 21, y Trome; además, dos diarios de cobertura regional (Correo y Ahora). Asimismo, se evaluaron las ediciones periodísticas de tres radiodifusoras de cobertura nacional con sus páginas webs: Radio Programas del Perú (RPP), Capital y Exitosa.

Se monitoreó los espacios periodísticos de cuatro canales de televisión de señal abierta de cobertura nacional: América TV, Latina TV, ATV, y Panamericana TV; y dos de señal cerrada (cable): RPP TV y Canal N.

Adicionalmente, se revisaron los medios de comunicación que tienen páginas web, de mayor aceptación en las regiones. En total se revisaron 60 páginas de entre radiodifusoras, diarios y portales web. Se consideró mínimo un medio de comunicación por región.

Asimismo, mediante la suscripción a alertas de buscadores como alertas google (https://www.google. com.pe/alerts) y promedmail (http://www.promedmail. org/) se fortaleció la detección de eventos que no eran informados primero por los medios de comunicación previamente seleccionados y monitoreados.

\section{CLASIFICACIÓN Y VERIFICACIÓN}

Las noticias se evaluaron individualmente, agrupando el titular, el cuerpo de la noticia (texto e imagen, audio y video), efectos/fondo musical y demás recursos informativos como una unidad de análisis.

La búsqueda y el registro (clasificación del tipo de evento, diagnóstico, función del medio y alcance) de las noticias fueron realizados por una comunicadora social, y la verificación de las noticias fue coordinada por un médico con el Equipo de Alerta Respuesta del CDC y las direcciones $u$ oficinas de epidemiología a nivel local (direcciones de redes integradas de salud [DISA]) y regional (direcciones regionales de salud [DIRESA] y gerencias regionales de salud [GERESA]).

El tipo de evento se clasificó en cuatro grupos $(8,9)$ : a) brote: el aumento inusual en el número de casos relacionados de un evento, de aparición súbita y diseminación localizada en un espacio específico, o cuando la nota periodística indicaba expresamente que se trataba de un brote; b) otras noticias de salud: todas las enfermedades que según los medios registraban un incremento significativo de casos, c) epizootia: equivalente a brote o epidemia de una enfermedad en una población animal, con la connotación de que puede afectar también a la población humana, d) evento de importancia para la salud pública (EVISAP): evento o daño extraordinario que pone en riesgo o afecta la seguridad sanitaria o la vida de la población humana. Se consideran a los accidentes de tránsito masivos, inundaciones, desbordes de ríos, deslizamientos, terremotos, incendios urbanos, contaminación por sustancias químicas, entre otros.

El diagnóstico o daño es el nombre de la enfermedad, epizootia y/o EVISAP del que informa la noticia. Se priorizó la búsqueda y registro de las enfermedades y eventos sujetos a vigilancia epidemiológica en el Perú (10).

La función del medio de comunicación (8,11,12) se categorizó, según lo descrito por Lasswell y Wright ${ }^{(12)}$ en: a) supervisión del entorno - circulación de noticias, a la que denominaremos informativa, y b) transmisión de cultura, a la que llamaremos educativa. Asimismo, se consideró la disfunción de la comunicación de masas de Charles R. Wright ${ }^{(12)}$, c) provocar pánico al informar sobre peligros, a la que denominaremos alarmista. Estas categorías se definieron de acuerdo a lo descrito en la Tabla 1.

El alcance de la noticia ${ }^{(11)}$ se definió en función al número de regiones que presentaron más eventos y tuvieron mayor exposición mediática en los medios de comunicación.

La verificación del evento sanitario (8) se realizó en coordinación con el nivel regional y/o local donde ocurrió el evento, para confirmarlo o descartarlo, clasificándolo de acuerdo a lo descrito también en la Tabla 1.

\section{ANÁLISIS DE DATOS}

Se realizaron los análisis de las frecuencias relativas de las características de los eventos (tipo de evento, tipo de diagnóstico/daño del evento, tipo de noticia, verificación del evento) registrados en la vigilancia de eventos sanitarios en medios de comunicación, de manera global y por años. Además, se mapearon los eventos confirmados.

\section{RESULTADOS}

Al final del periodo de estudio se logró recolectar un total de 1638 noticias entre los cuatro tipos de eventos sanitarios: brote, epizootia, EVISAP y otras noticias de salud. 
Tabla 1. Definiciones operacionales usadas para clasificar las noticias provenientes de medios de comunicación

\begin{tabular}{|c|c|}
\hline Tipo & Definición \\
\hline \multicolumn{2}{|l|}{ Noticia } \\
\hline Informativa & $\begin{array}{l}\text { La noticia informa el evento con objetividad, sin recurrir a textos, imágenes, audios y/o videos que } \\
\text { generen alarma, pánico, terror y/o temor }\end{array}$ \\
\hline Educativa & $\begin{array}{l}\text { La noticia difunde información para conocimiento de la enfermedad, aspectos preventivos y manejo } \\
\text { primario de esta. Asimismo, considera aspectos preventivos y de control sobre eventos de importancia } \\
\text { en salud pública }\end{array}$ \\
\hline Alarmista & $\begin{array}{l}\text { La noticia eleva infundadamente la percepción de riesgo de la población, al considerar audio y/o texto } \\
\text { equivalentes a palabras como "mortal", "maldita", "alarma", "pánico", "terror", "psicosis", "enfermedad } \\
\text { mortal", "plaga" y otros términos similares }\end{array}$ \\
\hline Informativa y educativa & La noticia cumple con las dos definiciones operativas de noticia informativa y noticia educativa. \\
\hline Alarmista y educativa & La noticia cumplió con las dos definiciones operativas de noticia alarmista y noticia educativa. \\
\hline \multicolumn{2}{|l|}{ Evento } \\
\hline Confirmado - notificado & $\begin{array}{l}\text { El evento sanitario fue notificado y/o fue de conocimiento del personal de salud del nivel regional y/o } \\
\text { local antes de ser difundido como noticia por un medio de comunicación. }\end{array}$ \\
\hline $\begin{array}{l}\text { Confirmado - no } \\
\text { notificado }\end{array}$ & $\begin{array}{l}\text { El evento sanitario es difundido inicialmente por un medio de comunicación, y después de la } \\
\text { investigación realizada por el personal de salud, el evento fue confirmado. }\end{array}$ \\
\hline $\begin{array}{l}\text { No concordante - } \\
\text { notificado }\end{array}$ & $\begin{array}{l}\text { Los datos del evento sanitario difundido por el medio de comunicación, fueron discordantes a los datos } \\
\text { registrados/notificados previamente por el personal de salud }\end{array}$ \\
\hline $\begin{array}{l}\text { No concordante - no } \\
\text { notificado }\end{array}$ & $\begin{array}{l}\text { Los datos del evento sanitario, difundido primero por un medio de comunicación, fueron discordantes a } \\
\text { los datos recogidos en el proceso de verificación/investigación }\end{array}$ \\
\hline Descartado & $\begin{array}{l}\text { La verificación y/o investigación determinó la no existencia del evento sanitario difundido por el medio } \\
\text { de comunicación. }\end{array}$ \\
\hline En investigación & $\begin{array}{l}\text { El proceso de verificación/investigación del evento difundido por el medio de comunicación seguía en } \\
\text { curso al cierre del año } 2015\end{array}$ \\
\hline
\end{tabular}

La Tabla 2 resume las características de los eventos registrados en la vigilancia de eventos sanitarios en medios de comunicación. Dentro de estas características destacó que el mayor porcentaje de noticias, respecto al tipo de eventos, correspondió a otras noticias de salud $(57,1 \%)$ y a EVISAP $(27,1 \%)$. Las enfermedades infecciosas de notificación obligatoria $(60,3 \%)$ representaron el tipo de diagnóstico/daño más frecuente, entre las que se encontraron dengue, enfermedades respiratorias y diarreicas, leptospirosis, entre otros; los desastres naturales $(12,8 \%)$ ocuparon el segundo lugar, entre los que destacaron los desbordes de ríos, huaicos, lluvias intensas, entre otros. Además, los accidentes de tránsito $(9,2 \%)$, ocuparon un lugar importante en la lista de la vigilancia de eventos sanitarios, al registrar de entre 10 muertos y/o 15 heridos por accidente.

Por otro lado, las noticias en su mayoría fueron de tipo informativo $(66,7 \%)$ evidenciando que en su mayoría los medios de comunicación estarían cumpliendo la función informativa, mientras que las noticias con tono alarmista fueron del 17,4\%; estas noticias son de importante observación porque son propensas a ocasionar pánico innecesario en la población debido a titulares como "Pánico por gripe A H1N1", "Otra epidemia", "Bebé contrajo temible bacteria en el Regional". Asimismo, las noticias alarmistas y educativas $(5,6 \%)$, aunque en menor porcentaje, han contribuido a elevar la percepción de riesgo de la población y, a su vez, brindaron información preventiva o educativa sobre el evento difundido. No se encontraron noticias en la categoría noticia educativa.

Esta vigilancia tiene un procedimiento de verificación de los eventos sanitarios. En ese sentido, las noticias confirmadas no notificadas $(37,9 \%)$ y no concordantes no notificadas $(11,5 \%)$ representaron en suma el $49,4 \%$ de eventos sanitarios que fueron difundidos primero por los medios de comunicación; por el contrario, los eventos que previamente fueron notificados representaron el $31,1 \%$; sin embargo, el $6,5 \%$ del total de eventos sanitarios registrados fueron descartados después de la investigación realizada por el personal de salud.

Con respecto a las fuentes de información, RPP fue el medio de comunicación que mayor noticias aportó a este subsistema de vigilancia con un $40,6 \%$ de las noticias, en segundo lugar se encontró el diario el Correo con un $20,6 \%$. Finalmente, los departamentos con mayor número de noticias fueron: La Libertad, Loreto, Junín y Piura.

En la Tabla 3 se detallan los eventos registrados en la vigilancia de eventos sanitarios en medios de comunicación por año. La proporción de eventos notificados por año fue variable, siendo el tipo de evento otras noticias de salud el más frecuentemente notificado en todos los años (más del $50 \%$ ), ocupando el segundo lugar los EVISAP. Durante el año 2013, los brotes alcanzaron mayor frecuencia a diferencia de los otros años, llegando a un 22,1\%. 
Tabla 2. Características de los eventos registrados en la vigilancia de eventos sanitarios en medios de comunicación, periodo 2013-2015

\begin{tabular}{|c|c|}
\hline $\begin{array}{l}\text { Características de los eventos } \\
\text { notificados }\end{array}$ & n (\%) \\
\hline \multicolumn{2}{|l|}{ Tipo de evento } \\
\hline Otras noticias de salud & $935(57,1)$ \\
\hline $\begin{array}{l}\text { Eventos de Importancia para la salud } \\
\text { pública (EVISAP) }\end{array}$ & $444(27,1)$ \\
\hline Brotes & $215(13,1)$ \\
\hline Epizootias & $44(2,7)$ \\
\hline \multicolumn{2}{|l|}{ Tipo de diagnóstico/daño del evento } \\
\hline $\begin{array}{l}\text { Enfermedad infecciosa de notificación } \\
\text { obligatoria }\end{array}$ & $988(60,3)$ \\
\hline Desastres naturales & $210(12,8)$ \\
\hline Accidente de tránsito & $151(9,2)$ \\
\hline Enfermedades transmitidas por alimentos & $87(5,3)$ \\
\hline Otras enfermedades & $58(3,5)$ \\
\hline Intoxicación por sustancias químicas & $42(2,6)$ \\
\hline Otros eventos & $33(2,0)$ \\
\hline Incendios en áreas urbanas & $29(1,8)$ \\
\hline Ofidismo & $12(0,7)$ \\
\hline $\begin{array}{l}\text { Eventos adversos posvacunación } \\
\text { (ESAVI) }\end{array}$ & $10(0,6)$ \\
\hline Loxocelismo & $9(0,6)$ \\
\hline Otras epizootias & $9(0,6)$ \\
\hline \multicolumn{2}{|l|}{ Tipo de noticia } \\
\hline Noticia informativa & $1093(66,7)$ \\
\hline Noticia alarmista & $285(17,4)$ \\
\hline Noticia informativa y educativa & $169(10,3)$ \\
\hline Noticia alarmista y educativa & $91(5,6)$ \\
\hline \multicolumn{2}{|l|}{ Verificación del evento sanitario } \\
\hline Confirmado - no notificado & $620(37,9)$ \\
\hline Confirmado - notificado & $378(23,1)$ \\
\hline En investigación & $215(13,1)$ \\
\hline No concordante - no notificado & $188(11,5)$ \\
\hline No concordante - notificado & $131(8,0)$ \\
\hline Descartado & $106(6,5)$ \\
\hline \multicolumn{2}{|l|}{ Fuentes de información (cobertura) } \\
\hline Radio Programas del Perú (nacional) & $665(40,6)$ \\
\hline Otras fuentes & $524(32,0)$ \\
\hline Diario Correo (nacional y regional) & $338(20,6)$ \\
\hline Diario Ahora (regional) & $63(3,9)$ \\
\hline Diario La República (nacional) & $48(2,9)$ \\
\hline \multicolumn{2}{|l|}{ Alcance de la noticia * } \\
\hline Otros & $724(44,2)$ \\
\hline La Libertad & $138(8,4)$ \\
\hline Loreto & $130(7,9)$ \\
\hline Junín & $129(7,9)$ \\
\hline Piura & $115(7,0)$ \\
\hline Arequipa & $109(6,7)$ \\
\hline Lima & $104(6,4)$ \\
\hline Ancash & $99(6,0)$ \\
\hline Puno & $90(5,5)$ \\
\hline
\end{tabular}

* Lugar de donde procede el evento.
De acuerdo a la clasificación de los eventos, los eventos confirmados pero no notificados, tuvieron mayor frecuencia que las otras categorías en todos los años estudiados. Además, se registró un 13,1\% como eventos que se encontraban en investigación y que no fueron clasificados al cierre del año. Durante los años 2014 y 2015, la proporción de eventos confirmados no notificados tuvieron una mayor frecuencia en la detección y verificación. En los tres años estudiados se registró un $37,9 \%$ de eventos confirmados no notificados y un $11,5 \%$ de eventos no concordantes no notificados, lo que en suma significa un $49,4 \%$ de eventos que no fueron identificados por los sistemas de vigilancia epidemiológica. Por otro lado, solo en el año 2013, la tercera parte de los eventos fueron confirmados y notificados, pero durante los años 2014 y 2015 , en suma, solo fue confirmado y notificado el $32,8 \%$ de eventos.

Las noticias informativas predominaron en todos los años con más del $60 \%$ de registros, mientras que las noticias alarmistas alcanzaron más del $15 \%$ durante tres los años estudiados.

Tabla 3. Características de los eventos registrados en la vigilancia de eventos sanitarios en medios de comunicación por año, 2013-2015.

\begin{tabular}{|c|c|c|c|}
\hline \multirow{2}{*}{ Evento } & 2013 & 2014 & 2015 \\
\hline & n (\%) & n (\%) & n (\%) \\
\hline \multicolumn{4}{|l|}{ Tipo de evento } \\
\hline $\begin{array}{l}\text { Otras noticias de } \\
\text { salud }\end{array}$ & $252(50,7)$ & $318(56,4)$ & $365(63,3)$ \\
\hline EVISAP & $125(25,2)$ & $181(32,1)$ & $138(23,9)$ \\
\hline Brotes & $110(22,1)$ & $48(8,5)$ & $57(9,9)$ \\
\hline Epizootias & $10(2,0)$ & $17(3,0)$ & $17(3,0)$ \\
\hline \multicolumn{4}{|l|}{$\begin{array}{l}\text { Clasificación del } \\
\text { evento }\end{array}$} \\
\hline $\begin{array}{l}\text { Confirmado - no } \\
\text { notificado }\end{array}$ & $193(38,8)$ & $224(39,7)$ & $203(35,2)$ \\
\hline $\begin{array}{l}\text { Confirmado - } \\
\text { notificado }\end{array}$ & $191(38,4)$ & $92(16,3)$ & $95(16,5)$ \\
\hline En investigación & $59(11,9)$ & $68(12,1)$ & $88(15,3)$ \\
\hline Descartado & $28(5,6)$ & $41(7,3)$ & $37(6,4)$ \\
\hline $\begin{array}{l}\text { No concordante - } \\
\text { no notificado }\end{array}$ & $16(3,2)$ & $88(15,6)$ & $84(14,6)$ \\
\hline $\begin{array}{l}\text { No concordante - } \\
\text { notificado }\end{array}$ & $10(2,0)$ & $51(9,0)$ & $70(12,1)$ \\
\hline \multicolumn{4}{|l|}{ Tipo de noticia } \\
\hline Noticia informativa & $366(73,6)$ & $355(62,9)$ & $372(64,5)$ \\
\hline Noticia alarmista & $94(18,9)$ & $90(16)$ & $101(17,5)$ \\
\hline $\begin{array}{l}\text { Noticia informativa } \\
\text { y educativa }\end{array}$ & $24(4,8)$ & $76(13,5)$ & $69(12,0)$ \\
\hline $\begin{array}{l}\text { Noticia alarmista y } \\
\text { educativa }\end{array}$ & $13(2,6)$ & $43(7,6)$ & $35(6,1)$ \\
\hline
\end{tabular}

EVISAP: Eventos de importancia para la salud pública. 
Tabla 4. Características de los diagnósticos por tipo de evento registrados en la vigilancia de eventos sanitarios en medios de comunicación por año, 2013-2015

\begin{tabular}{|c|c|c|c|}
\hline \multirow{2}{*}{ Diagnóstico de evento* } & 2013 & 2014 & 2015 \\
\hline & n (\%) & n (\%) & n (\%) \\
\hline \multicolumn{4}{|l|}{ Brote } \\
\hline Carbunco & $2(1,7)$ & $0(0,0)$ & $1(1,4)$ \\
\hline Chinkungunya & $0(0,0)$ & $0(0,0)$ & $2(2,7)$ \\
\hline Dengue & $13(10,8)$ & $4(6,2)$ & $7(9,5)$ \\
\hline Enfermedades transmitidas por alimentos & $46(38,3)$ & $21(32,3)$ & $20(27,0)$ \\
\hline Enfermedades diarreicas agudas & $3(2,5)$ & $3(4,6)$ & $0(0,0)$ \\
\hline Fiebre amarilla & $4(3,3)$ & $0(0,0)$ & $3(4,1)$ \\
\hline Infecciones intrahospitalarias & $4(3,3)$ & $3(4,6)$ & $5(6,8)$ \\
\hline Influenza A H1N1 & $2(1,7)$ & $2(3,1)$ & $2(2,7)$ \\
\hline Leishmaniasis & $1(0,8)$ & $0(0,0)$ & $1(1,4)$ \\
\hline Leptospirosis & $1(0,8)$ & $2(3,1)$ & $1(1,4)$ \\
\hline Malaria & $4(3,3)$ & $1(1,5)$ & $2(2,7)$ \\
\hline Meningitis & $2(1,7)$ & $2(3,1)$ & $1(1,4)$ \\
\hline Otras enfermedades & $16(13,3)$ & $7(10,6)$ & $8(10,8)$ \\
\hline Peste & $4(3,3)$ & $1(1,5)$ & $1(1,4)$ \\
\hline Rabia humana silvestre & $3(2,5)$ & $1(1,5)$ & $1(1,4)$ \\
\hline Sarampión & $0(0,0)$ & $0(0,0)$ & $2(2,7)$ \\
\hline Tuberculosis & $5(4,2)$ & $1(1,5)$ & $0(0,0)$ \\
\hline \multicolumn{4}{|l|}{ Epizootia } \\
\hline Carbunco & $0(0,0)$ & $1(1,5)$ & $1(1,4)$ \\
\hline Otras epizootias & $2(1,7)$ & $6(9,2)$ & $1(1,4)$ \\
\hline Peste & $1(0,8)$ & $0(0,0)$ & $0(0,0)$ \\
\hline Rabia animal & $7(5,8)$ & $10(15,4)$ & $15(20,3)$ \\
\hline \multicolumn{4}{|l|}{ Otras Noticias de Salud } \\
\hline Carbunco & $0(0,0)$ & $0(0,0)$ & $4(1,1)$ \\
\hline Chinkungunya & $0(0,0)$ & $4(1,3)$ & $29(8,0)$ \\
\hline Dengue & $52(20,6)$ & $90(28,3)$ & $138(37,8)$ \\
\hline Enfermedades diarreicas agudas & $8(3,2)$ & $15(4,7)$ & $6(1,6)$ \\
\hline ESAVI & $6(2,4)$ & $4(1,3)$ & $0(0,0)$ \\
\hline Hepatitis B & $5(2)$ & $2(0,6)$ & $2(0,6)$ \\
\hline Infecciones intrahospitalarias & $2(0,8)$ & $0(0,0)$ & $3(0,8)$ \\
\hline Infecciones respiratorias agudas & $15(6,0)$ & $29(9,1)$ & $25(6,9)$ \\
\hline Influenza A H1N1 & $82(32,5)$ & $43(13,5)$ & $21(5,8)$ \\
\hline Intoxicación por sustancia químicas & $6(2,4)$ & $14(4,4)$ & $22(6,0)$ \\
\hline Leishmaniasis & $1(0,4)$ & $7(2,2)$ & $3(0,8)$ \\
\hline Leptospirosis & $7(2,8)$ & $7(2,2)$ & $2(0,6)$ \\
\hline Loxocelismo & $3(1,2)$ & $2(0,6)$ & $4(1,1)$ \\
\hline Malaria & $7(2,8)$ & $11(3,5)$ & $5(1,4)$ \\
\hline Muerte materna & $4(1,6)$ & $8(2,5)$ & $23(6,3)$ \\
\hline Neumonía & $23(9,1)$ & $28(8,8)$ & $32(8,8)$ \\
\hline Ofidismo & $1(0,4)$ & $5(1,6)$ & $6(1,6)$ \\
\hline Otras enfermedades & $20(8,0)$ & $27(8,5)$ & $20(5,5)$ \\
\hline Peste & $4(1,6)$ & $3(0,9)$ & $0(0,0)$ \\
\hline Rabia humana urbana & $0(0,0)$ & $2(0,6)$ & $6(1,6)$ \\
\hline Tuberculosis & $6(2,4)$ & $16(5,0)$ & $14(3,8)$ \\
\hline \multicolumn{4}{|l|}{ EVISAP } \\
\hline Accidente de transito & $38(30,4)$ & $66(36,5)$ & $47(34,1)$ \\
\hline Desborde de río & $24(19,2)$ & $31(17,1)$ & $19(13,8)$ \\
\hline Deslizamiento & $5(4,0)$ & $9(5,0)$ & $3(2,2)$ \\
\hline Huaico & $6(4,8)$ & $7(3,9)$ & $8(5,8)$ \\
\hline Incendio urbano & $12(9,6)$ & $7(3,9)$ & $10(7,3)$ \\
\hline Inundación & $9(7,2)$ & $17(9,4)$ & $7(5,1)$ \\
\hline Lluvias intensas & $10(8,0)$ & $12(6,6)$ & $24(17,4)$ \\
\hline \multicolumn{4}{|l|}{ Otros eventos } \\
\hline Sismo & $5(4,0)$ & $10(5,5)$ & $3(2,2)$ \\
\hline Vientos fuertes & $4(3,2)$ & $5(2,8)$ & $13(9,4)$ \\
\hline
\end{tabular}

EVISAP: Eventos de importancia para la salud pública.

ESAVI: Eventos adversos posvacunación.

* Tipos de eventos ordenados por orden alfabetico 
Entre los brotes registrados, con respecto al diagnóstico del evento, las enfermedades transmitidas por alimentos (ETA) fueron las que tuvieron mayor frecuencia en los tres años de estudio, en segundo y tercer lugar destacaron los brotes de dengue e infecciones intrahospitalarias. En lo que respecta a las epizootias, el registro de casos de rabia animal fue incrementándose año tras año. En otras noticias de salud destacaron el dengue, influenza A H1N1 y las neumonías como las que tuvieron mayor frecuencia de registro durante todos los años. Es importante destacar que durante el 2013, la influenza A H1N1 alcanzó su mayor frecuencia llegando hasta $32,5 \%$. En ese orden, los accidentes de tránsito y los desbordes de los ríos fueron los EVISAP que tuvieron mayor frecuencia de aparición en los medios de comunicación durante los tres años.

En la Figura 1, se muestra la distribución espacial de los eventos confirmados. Por ejemplo los brotes confirmados fueron más frecuentes en Loreto, Ucayali, Lima, Ancash y La Libertad. Las epizootias confirmadas fueron más frecuentes en Arequipa. Los EVISAP confirmados fueron más frecuentes en Lima, Ancash, Arequipa, Junín, Huancavelica y San Martín. Los otros eventos confirmados fueron más frecuentes en Loreto, Piura, Junín y La Libertad.

\section{DISCUSIÓN}

La vigilancia de eventos sanitarios es una estrategia importante de la vigilancia basada en la notificación de casos, porque permite reconocer eventos que no son identificados por los sistemas de vigilancia tradicionales, principalmente eventos de poca magnitud como brotes de ETA o el inicio de epidemias, entre otros ${ }^{(5,6,13,14)}$.

Nuestro estudio confirma la importancia de la vigilancia de eventos sanitarios, ya que permitió identificar, desde el 2013 al 2015, el 49,4\% de eventos (confirmados no notificados y no concordantes no notificados) que fueron difundidos primero por los medios de comunicación y luego verificados y confirmados por los sistemas de vigilancia epidemiológica regular a nivel local, regional o nacional.

La utilidad de estos sistemas ha sido reconocida en otros estudios, como el estudio realizado por Schwind et al. ${ }^{(15)}$ en cinco países, donde usaron la herramienta Healthmap (que busca en internet eventos de salud) y una estrategia de vigilancia de los medios de comunicación locales; los investigadores lograron identificar 87 y 236 eventos de importancia en salud, respectivamente. Además, el estudio realizado por Gluskin et al. ${ }^{(16)}$ en 17 estados de México, demostró que la búsqueda de eventos de salud en el internet, como casos de dengue, es una herramienta
A

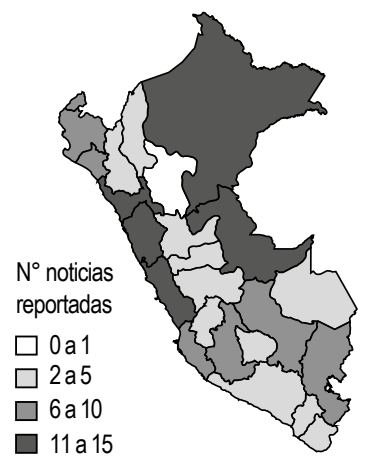

C

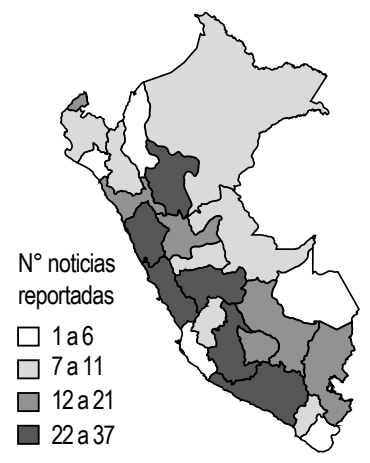

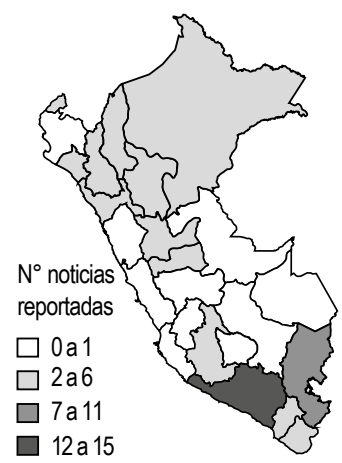

D

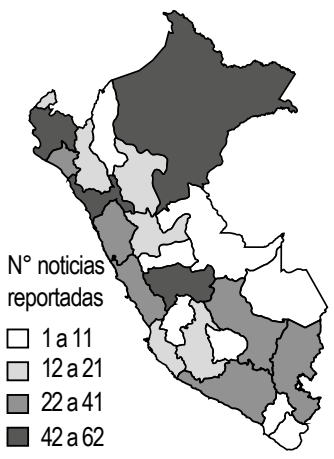

Figura 1. Distribución geográfica de A) brotes, B) Epizootias, C) EVISAP y D) otros 
que proporciona información rápida, que complementa los sistemas de vigilancia epidemiológica.

Por otro lado, la vigilancia de eventos sanitarios en medios de comunicación que conduce el CDC del MINSA ha contribuido a mejorar la respuesta a los brotes, epizootias y EVISAP, porque ha promovido que el nivel local y regional investigue un importante número de eventos no notificados por los sistemas regulares. La mejora en la respuesta frente a brotes y eventos de importancia en salud pública, también ha sido señalado en otros estudios y por OMS ${ }^{(1,17)}$, por ejemplo, han servido para desencadenar alertas sobre brotes de influenza, o potenciales emergencias de salud pública ${ }^{(5)}$, que han activado la respuesta rápida de los servicios de salud pública. En este sentido, estos sistemas han permitido mejorar la respuesta y el control de estos eventos y, por ende, contribuyen al mejoramiento de la salud pública, como se ha mencionado en algunas revisiones sistemáticas de otros sistemas basados en medios de comunicación y redes sociales ${ }^{(15,17)}$. A pesar de estos beneficios identificados en nuestro estudio y en otros estudios, todavía este tipo de vigilancia no está ampliamente integrado en los sistemas de vigilancia epidemiológica de todos los países.

Esta vigilancia permite, además, valorar el contenido de la noticia, que es muy importante para la comunicación de riesgo, porque permite monitorizar como informan los medios de comunicación sobre brotes y eventos de importancia en salud pública, e identificar noticias que elevan innecesariamente la percepción de riesgo frente a determinados eventos, generando así alarma y desesperación en la población ${ }^{(11)}$. Este estudio identificó que el $17,4 \%$ de las noticias analizadas fueron alarmistas, estas noticias alarmistas se dan principalmente durante brotes y/o epidemias, por ejemplo, en el caso de nuestro país, epidemias de dengue, o durante o pospandemias de influenza, etc. ${ }^{(11)}$. En este sentido, el MINSA tiene que monitorizar estas noticias con la finalidad de tomar acciones para reducir el impacto de estas noticias a través de la comunicación de riesgo, además de evaluar el trabajo de acercamiento que se hace con los medios de comunicación ${ }^{(3)}$.

Las vigilancias basadas en medios de comunicación o redes sociales, son una herramienta altamente eficaz, sensible, de bajo costo y sostenible en el tiempo, para la detección de brotes ${ }^{(18)}$. En este sentido, esta vigilancia que conduce el CDC del MINSA se debe fortalecer y, sobre todo, usar la información que brinda con el fin de mejorar las acciones que se ejecutan para mejorar salud pública del país, acelerar la respuesta a brotes y eventos de importancia en salud pública y así reducir su impacto en la población.

Contribuciones de autoría: los autores AP y CM participaron en la concepción y diseño del estudio. AP participó en la recolección de datos. CM participó en el análisis e interpretación de datos, AP y CM participaron en la redacción del manuscrito, en la revisión crítica del manuscrito y aprobaron su versión final.

Agradecimientos: al Centro Nacional de Epidemiología, Prevención y Control de Enfermedades (CDC) por el apoyo incondicional a esta investigación al brindar las facilidades del caso para realizarla. A la médica cirujana, Milagros Durand y al Equipo de Alerta-Respuesta del CDC por la verificación de los eventos sanitarios; y a las DIRESA Ayacucho, Loreto y San Martín por su apoyo en la vigilancia de eventos sanitarios en medios de comunicación.

Fuentes de financiamiento: autofinanciado.

Conflictos de interés: los autores declaran no tener conflictos de interés en la publicación de este artículo.

Renuncia de responsabilidad: los contenidos de este manuscrito solamente son responsabilidad de los autores y no necesariamente representan la opinión oficial del CDC del MINSA.

\section{REFERENCIAS BIBLIOGRÁFICAS}

1. World Health Organization (WHO). Early detection, assessment and response to acute public health events: Implementation of Early Warning and Response with a focus on Event-Based Surveillance, Interim Version. Ginebra: WHO; 2014

2. World Health Organization (WHO). International health regulations (2005). Ginebra: WHO; 2006

3. Organización Mundial de la Salud (OMS). La comunicación de brotes epidémicos "Prácticas óptimas para la comunicación con el público durante un brote epidémico. Singapur: OMS; 2014.
4. Hartley DM. Using social media and internet data for public health surveillance: the importance of talking. Milbank Q. 2014;92(1):34-9. doi: 10.1111/1468-0009.12039

5. Milinovich GJ, Williams GM, Clements AC, $\mathrm{Hu}$ W. Internet-based surveillance systems for monitoring emerging infectious diseases. Lancet Infect Dis. 2014;14(2):160-8. doi: 10.1016/S14733099(13)70244-5

6. Velasco E, Agheneza T, Denecke K, Kirchner G, Eckmanns T. Social media and internet-based data in global systems for public health surveillance: a systematic review. Milbank Q. 2014;92(1):7-33. doi: 10.1111/1468-0009.12038

7. Centro Nacional de Epidemiología, Prevención y Control de Enfermedades (CDC) [Internet]. Lima, Perú: CDC; c2005-2017. Sistema de alerta respuesta [citado el 15 de diciembre 2016]; [una página]. Disponible en: http://www.dge. gob.pe/portal/index.php?option $=$ com content $\&$ view $=$ article $\& i d=408 \&$ Itemid $=197$

8. Dirección General de Epidemiología (DGE). Sistema de alerta y respuesta: modelo de vigilancia de rumores. Lima, Perú: Ministerio de Salud del Perú; 2013. 
9. Perú,Dirección GeneraldeEpidemiología (DGE). Directiva sanitaria de notificación de brotes, epidemias y otros eventos de importancia para la salud pública. D.S. No 047-MINSA/DGE-V.01. Lima: DGE; 28 de junio del 2012.

10. Perú, Dirección General de Epidemiología (DGE). Directiva sanitaria de notificación de enfermedades y eventos sujetos a vigilancia epidemiológica en salud pública. D.S. No 046-MINSA/DGE-V.01. Lima: DGE, 18 de junio del 2012.

11. Palpan-Guerra A, Munayco CV. ¿Cómo informaron los medios de comunicación sobre la influenza AH1N1 en Perú? Rev Peru Med Exp Salud Pública. 2015;32(2):294-8.

12. Lozano J. Teoría e investigación de la comunicación de masas. 2da ed. México: Pearson Educación de México; 2007.

13. Meng L, Lyu Y, Cao Y, Tu W, Hong Z, Li L, et al. [Information obtained through Internet-based media surveillance regarding domestic public health emergencies in
2013]. Zhonghua Liu Xing Bing Zue Za Zhi. 2015;36(6):607-11. Chino.

14. Elliot AJ, Hughes HE, Astbury J, Nixon G, Brierley K, Vivancos R, et al. The potential impact of media reporting in syndromic surveillance: an example using a possible Cryptosporidium exposure in North West England, August to September 2015. Euro Surveill. 2016;21(41):pii=30368. doi: 10.2807/1560-7917.ES.2016.21.41.30368

15. Schwind JS, Wolking DJ, Brownstein JS, Mazet JA, Smith WA. Evaluation of local media surveillance for improved disease recognition and monitoring in global hotspot regions. PloS One. 2014;9(10):e110236. doi: 10.1371/journal.pone.0110236

16. Gluskin RT, Johansson MA, Santillana M, Brownstein JS. Evaluation of Internet-based dengue query data: Google Dengue Trends. PLoS Negl Trop Dis. 2014;8(2):e2713. doi: 10.1371/journal. pntd.0002713

17. Charles-Smith LE, Reynolds TL, Cameron MA, Conway M, Lau EH, Olsen JM, et al. Using social media for actionable disease surveillance and outbreak management: a systematic literature review. PloS One. 2015;10(10):e0139701. doi: 10.1371/journal.pone.0139701

18. Ao TT, Rahman M, Haque F, Chakraborty A, Hossain MJ, Haider $\mathrm{S}$, et al. Low-Cost National MediaBased Surveillance System for Public Health Events, Bangladesh. Emerg Infect Dis. 2016;22(4):720-2. doi: 10.3201/ eid2204.150330

Correspondencia: Lic. Ada Palpán Guerra Dirección: Av. Alfredo Mendiola 6821 Condominio Las Torres Block " $X$ ", Dpto. 904, Los Olivos.

Teléfono: (+51) 945036357

Correo electrónico: adapalpan@gmail.com

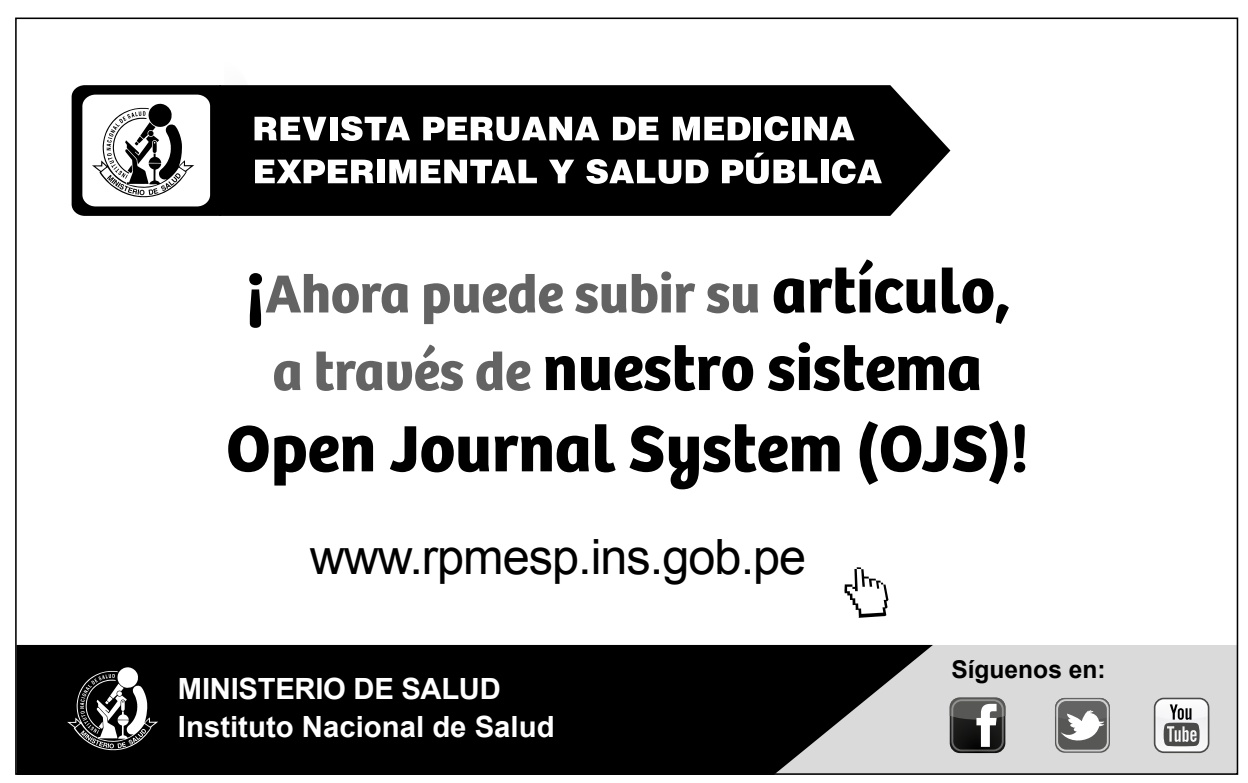

